\title{
A Proposal for an Asymmetric Best-Effort Service
}

\author{
Paul Hurley <paul.hurley@epfl.ch>, Jean-Yves Le Boudec <leboudec@di.epfl.ch> \\ ICA, Ecole Polytechnique Fédérale de Lausanne (EPFL).
}

\begin{abstract}
We propose Asymmetric Best-Effort, a novel service to provide a "throughput versus delay jitter" differentiated service for IP packets. With this service, every best effort packet is marked as either Green or Blue. Green packets, typically sent by real-time applications such as interactive audio, receive more losses during bouts of congestion than Blue ones. In return, they receive less delay jitter.

Both Green and Blue services are best-effort. The incentive to choose one or other is based on the nature of one's traffic and on traffic conditions. If applications are TCP-friendly, those sending Blue packets will receive more throughput but also more delay jitter, than they would if they sent Green packets for a given network state and path.
\end{abstract}

\section{Introduction}

We propose a new Asymmetric Best-Effort service. It partitions IP packets into Green packets, which are given low delay jitter guarantees through the network without reservation, and Blue packets which receive fewer losses during bouts of congestion than Green ones.

Provided sources are "TCP-friendly", those who choose to be Blue receive higher throughput than they would if they had chosen to be Green. It is important to emphasise that these are both best-effort services, and the incentive to choose one or other is based on the nature of one's traffic with overall benefit for both traffic types.

Each packet is either Green or Blue. Green packets would usually be interactive traffic such as Internet Telephony where packet transfer from end to end must be short and delay jitter significant. Blue packets are typically non-interactive traffic such as TCP traffic whose end to end delay can be variable and the goal is minimisation of overall transfer time. We leave open to subsequent definition how the Green and Blue distinction should be made.

The amount of negative feedback (e.g. packet losses) received by Green traffic is greater than that received by Blue traffic. The admitted Green packets are given a shorter queueing delay. The network-level quality of service, packet loss and delay jitter, received by one of the traffic types cannot be classified as being better than the other.

No rate reservation is assumed. During a silent period of a given traffic type, the other can make use of the whole bandwidth. Traffic management and charging practices remain essentially the same as for a single class best-effort network. Unsatisfactory trade-offs between the different buffer size requirements of real and non-real time traffic can also be avoided.

We assume that Green and Blue sources are "TCPfriendly" [1], i.e. they do not send more than a TCP source would for the same conditions of loss. Although the approach described remains valid when using some form of explicit negative feedback such as Explicit Congestion Notification (ECN), we consider here only the case where packet loss implicitly provides feedback.

\section{Router Implementation}

Asymmetric Best-Effort traffic control can be supported within an IP router by Packet Admission Control (PAC) and differential scheduling. The PAC manages the queue by dropping packets whenever necessary or appropriate, acceptance being biased in favour of Blue packets. The scheduler determines which packet, if any, from the buffer should be sent next, with bias in favour of Green packets.

We now describe one router implementation which was inserted and simulated in ns [5]. The service goal is the distribution of throughput such that a Blue flow would receive approximately $\beta$ times as much throughput as a Green one that shares the same path.

The scheduling is Earliest Deadline First (EDF) [4]. Each packet is assigned a finishing service time deadline, a tag, and the packet currently having the lowest value is served first (i.e. earliest deadline).

Each Green packet arriving is assigned a finishing service time deadline equal to the arrival time $t$. A Blue packet is assigned a time equal to the arrival time plus a constant $D$, namely $t+D$. Using EDF, rather than a plain priority scheme in which Green packets would always be served first, has the advantage of preventing service starvation for Blue traffic. 


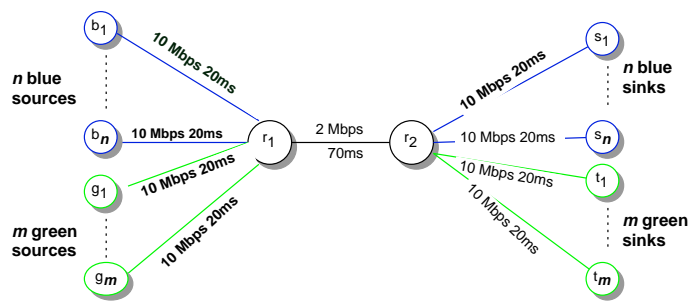

Figure 1: Simulation Network used in Illustration of Service

The PAC comprises a modified version of Random Early Detection (RED) [3] in which the dropping probability for Blue packets is the usual RED dropping probability $p$ while for Green packets it is $\alpha p$.

In addition, to ensure Green packets are given a sufficiently small delay they must pass a second acceptance decision, called Green delay control. For this implementation, a Green packet is accepted into the queue only if the number of packets, $N$, in the queue with tag of less than the current time, is less than or equal to a given system permitted number $N_{g}$. The PAC algorithm can now be summarised as follows:

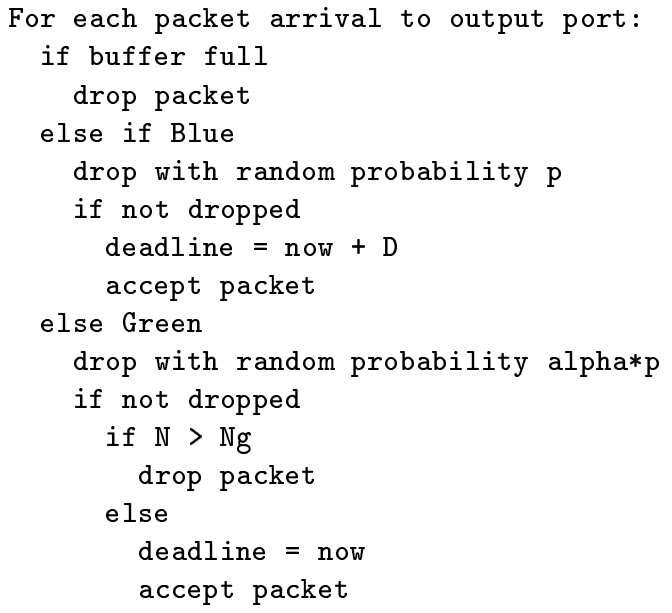

$\alpha$ is varied such that it drives the effective measured ratio of Green to Blue drop ratios $\alpha_{m}$ towards the target ratio of Green to Blue loss ratio $\alpha_{t}$. For this iteration, the mechanism to drive $\alpha_{m}$ towards $\alpha_{t}$ is simple and given by $\alpha=\alpha_{t}^{2} / \alpha_{m} . \alpha_{t}$ is chosen to be of the order of $\beta^{2}$, as described in [2], in order to attain the service goal. As ongoing work, the target ratio $\alpha$ is currently being adjusted to allow for the fact that the lower queueing delay causes Green flows to have a lower round-trip time and thus achieve a higher throughput than one expects if the round-trip time was the same.

\section{Service Illustration}

We show that an application that requires low delay jitter and thus sends Green packets does receive it but

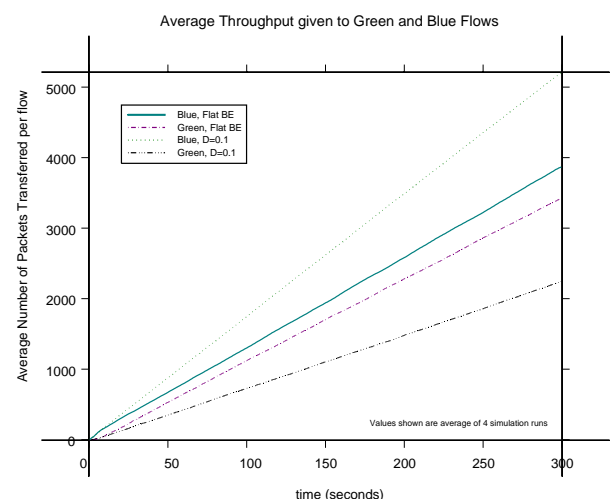

Figure 2: Average of the average number of packets received per Green and Blue flow with/without Asymmetric Best-Effort $(n=m=10)$.

at the expense of lower throughput. Conversely, an application that does not care about jitter receives overall reduced end to end transfer delay.

The network used in this simulation is shown in Figure 1. $n$ Blue and $m$ Green flows share a bottleneck and the same nonqueueing delay. Router $r_{1}$ facilitates the service by the implementation as described in Section 2. The Blue sources are TCP Reno.

Since a Green source would typically be rate rather than window based, and not necessarily concerned with loss recovery given its real-time nature, it uses a simple transport protocol designed to approximate TCP friendliness as described in [2].

For all simulation results shown, $D=0.1, \beta=$ 3 and $N_{g}=10$. Packet sizes were a constant 1000 bytes, and the buffer size at $r_{1}$ was 60 packets. The RED parameters chosen were $\min _{t h}=0, \max _{t h}=40$, $\max _{p}=0.2$ and $w=0.02$ which are not the usual range of parameters used. With more typical RED parameters there are many forced losses and hence less control over the dropping differential.

The first simulation results we show are for $n=$ 10 and $m=10$. Figure 2 shows the average over 4 simulation runs of the average number of packets transferred by each traffic type at a given time $t$ for the cases with and without Asymmetric Best-Effort. The throughput given to Blue traffic is clearly higher in the asymmetric case. The worst-case interval for $95 \%$ confidence was 1.30 packets. Figure 3 shows the better distribution of queueing delays experienced by Green traffic at router $r_{1}$ when using Asym Best-Effort.

Overall benefit for each source is achieved. A source that sees value in low delay jitter will choose to be Green. This enables file transfer oriented applications, which choose to be Blue, to achieve higher throughput. 


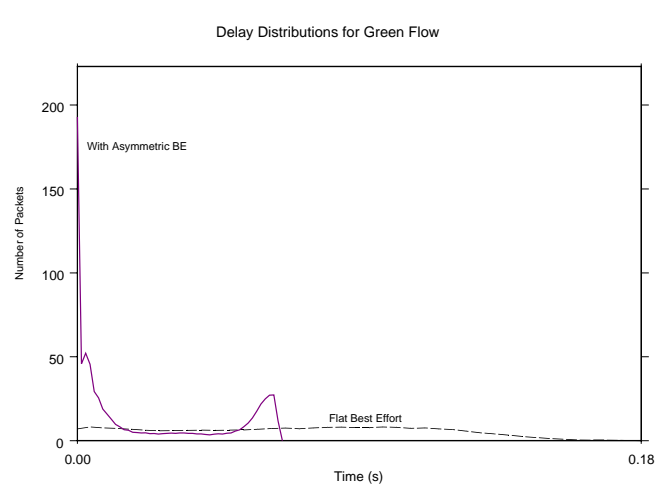

Figure 3: Queueing Delay for Green packets with/without Asymmetric Best-Effort. $(n=m=10)$.

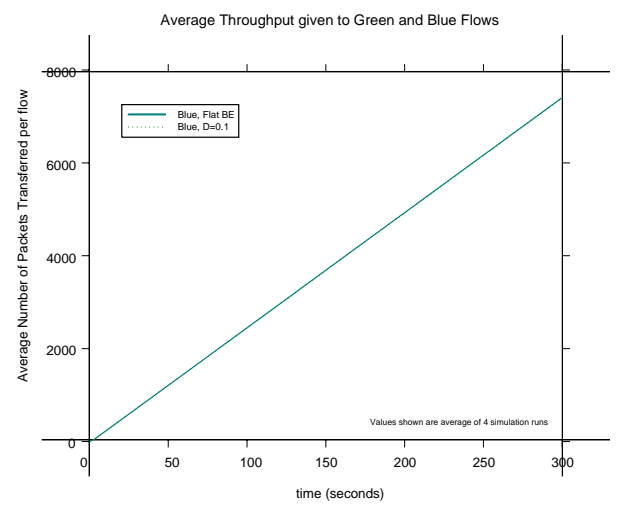

Figure 4: With all Blue traffic, Asymmetric BestEffort resorts to the flat case.

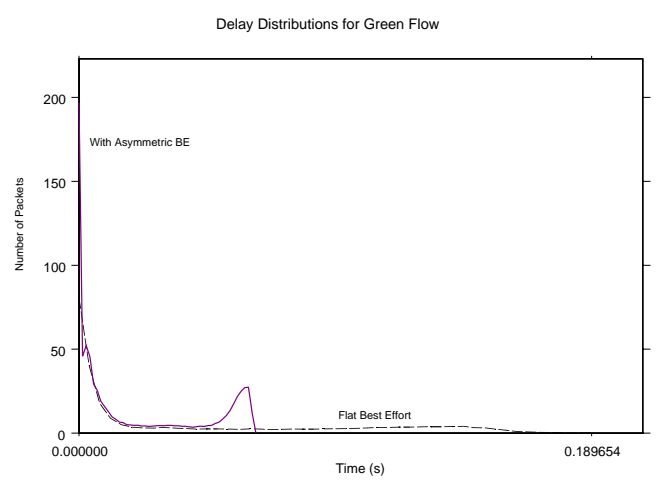

Figure 5: If all Green traffic, a lower delay with Asymmetric Best-Effort is still received.
Figure 4 shows, in the case of all-Blue traffic ( $n=$ $10, m=0$ ), that the service reverts to regular flat besteffort. The worst-case interval for $95 \%$ confidence here was 0.3561 packets.

Figure 5 shows, in the case of all-Green traffic ( $n=$ $0, m=10)$, the queueing delay distribution for Green traffic for the service is still better than in flat besteffort, due to the effect of the green dropping control, which permits only a small number of green packets in the queue.

\section{Conclusions}

We have described a simple but powerful service which enables best-effort traffic to receive requirements closer to its traffic desires yet to everyone's overall benefit. It decouples delay jitter objectives from loss objectives with no concept of reservation or signalling and no change to traffic management or charging. Dimensioning the network is also potentially simpler since one would no longer need to choose a buffering compromise to suit both types of traffic.

It should be stressed that Asymmetric Best-Effort is a new service in its own right and not a substitute for reservation or priority services.

The service choice of Green or Blue is self-policing since the user/application will be coaxed into choosing one or the other or indeed a mixture of both, based on its traffic profile objectives.

We provided a first implementation of the service, of which further optimisation of the control loop and the setting of RED parameters remains. In particular, the dropping probability for Green packets needs to be increased to compensate for the shorter queueing delay resulting in higher throughput.

\section{References}

[1] TCP friendly web site. http://www.psc.edu/networking/tcp_friendly.html

[2] Paul Hurley, Jean-Yves Le Boudec, Maher Hamdi, Ljubica Blazevic, Patrick Thiran. The Asymmetric Best-Effort Service. SSC Technical Report SSC/1999/003, EPFL, February 1999.

[3] Floyd, S., and Jacobson, V. Random Early Detection gateways for Congestion Avoidance. IEEE/ACM Transactions on Networking, V.1 N.4, August 1993, p.397-413.

[4] R. Guerin and V. Peris. Quality-of-Service in Packet Networks - Basic Mechanisms and Directions. Computer Networks and ISDN Systems. Special issue on multimedia communications over packet based networks, 1998.

[5] ns v2 simulator. See http://wwwmash.cs.berkeley.edu/ns / 